\title{
Is Lavelle-McMullan transformation a really new symmetry in QED?
}

\author{
D. K. Park \\ Department of Physics, KyungNam University, Masan, 631-701 Korea \\ Hung Soo Kim and Jae Kwan Kim \\ Department of Physics, KAIST, Taejon, 305-701, Korea
}

\begin{abstract}
Lavelle-McMullan symmetry of QED is examined at classical and quantum levels. It is shown that Lavelle-McMullan symmetry does not give any new non-trivial information in QED by examining the Ward-Takahashi identities. Being inspired by the examination of Ward-Takahashi identity, we construct the generalized non-local and non-covariant symmetries of QED.
\end{abstract}


In a recent work Lavelle-McMullan(LM) found a non-local and noncovariant symmetry of QED in the Feynman gauge[1]. They claimed that this is new symmetry and the physical states of QED are restricted by the conditions

$$
\begin{aligned}
Q_{B R S T} \mid \text { phys }> & =0, \\
Q_{L M} \mid \text { phys }> & =0,
\end{aligned}
$$

where $Q_{B R S T}$ and $Q_{L M}$ are charges of BRST[2,3] and LM transformations respectively. More recently it was disputed whether LM symmetry is merely non-local version of BRST symmetry or not $[4,5]$.

In the present paper we will examine this subject thoroughly by deriving the Ward-Takahashi(WT) identities and show that LM symmetry does not yield any new non-trivial information. In addition we will construct the generalized non-local and non-covariant symmetry for QED.

The QED Lagrangian we will study in Feynman gauge is

$$
\mathcal{L}=-\frac{1}{4} F_{\mu \nu} F^{\mu \nu}-\frac{1}{2}\left(\partial_{\mu} A^{\mu}\right)^{2}+\bar{\psi}\left(i \gamma^{\mu} D_{\mu}-m\right) \psi-i \partial_{\mu} \bar{c} \partial^{\mu} c
$$

where $D_{\mu}=\partial_{\mu}+i g A_{\mu}$. The BRST symmetry for the Lagrangian (2)

$$
\begin{aligned}
\delta_{B R S T} A_{\mu} & =\partial_{\mu} c \\
\delta_{B R S T} c & =0 \\
\delta_{B R S T} \bar{c} & =-i \partial_{\mu} A^{\mu} \\
\delta_{B R S T} \psi & =-i g c \psi \\
\delta_{B R S T} \bar{\psi} & =-i g \bar{\psi} c
\end{aligned}
$$

yields a conserved current through Noether's theorem

$$
J_{B R S T}^{\mu}=-\left(F^{\mu \nu}+g^{\mu \nu} \partial_{\rho} A^{\rho}\right) \partial_{\nu} c+g J^{\mu} c,
$$


where $J^{\mu}=\bar{\psi} \gamma^{\mu} \psi$.

On the other hand LM symmetry

$$
\begin{aligned}
\delta_{L M} A_{0} & =i \bar{c} \\
\delta_{L M} A_{i} & =i \frac{\partial_{i} \partial_{0}}{\nabla^{2}} \bar{c}, \\
\delta_{L M} c & =A_{0}-\frac{\partial_{i} \partial_{0}}{\nabla^{2}} A_{i}+\frac{g}{\nabla^{2}} J_{0}, \\
\delta_{L M} \bar{c} & =0, \\
\delta_{L M} \psi & =\left(\frac{g}{\nabla^{2}} \partial_{0} \bar{c}\right) \psi, \\
\delta_{L M} \bar{\psi} & =\bar{\psi}\left(\frac{g}{\nabla^{2}} \partial_{0} \bar{c}\right),
\end{aligned}
$$

yields another conserved current

$$
\begin{aligned}
J_{L M}^{\mu}= & -i\left(F^{\mu 0}+g^{\mu 0} \partial_{\rho} A^{\rho}\right) \bar{c}-i\left(F^{\mu i}+g^{\mu i} \partial_{\rho} A^{\rho}\right) \frac{\partial_{i} \partial_{0}}{\nabla^{2}} \bar{c} \\
& +i J^{\mu}\left(\frac{g}{\nabla^{2}} \partial_{0} \bar{c}\right)+i \square \bar{c} \frac{F^{0 \mu}}{\nabla^{2}} .
\end{aligned}
$$

Using equations of motion

$$
\begin{aligned}
g J^{\nu} & =\partial_{\mu}\left(F^{\mu \nu}+g^{\mu \nu} \partial_{\rho} A^{\rho}\right), \\
\left(i \gamma^{\mu} D_{\mu}-m\right) \psi & =0, \\
\square c=\square \bar{c} & =0,
\end{aligned}
$$

one can straightforwardly prove

$$
\partial_{\mu} J_{L M}^{\mu}=i c^{-1}\left(\frac{\partial_{0}}{\nabla^{2}} \bar{c}\right) \partial_{\mu} J_{B R S T}^{\mu} .
$$

Eq.(8) implys that BRST symmetry guarantees the symmetric property of LM transformation even at the classical level. This means LM symmetry is not independent of BRST symmetry. 
In order to study the quantum theory let us examine the WT identities. Taking a LM variation to the well-known identities of QED

$$
<0\left|T A_{\mu}(x) c(y)\right| 0>=0
$$

one gets

$$
\begin{array}{r}
-i D(q)+D_{00}(q)-\frac{q_{i} q_{0}}{\vec{q}^{2}} D_{0 i}(q)-\frac{g}{\vec{q}^{2}} \Lambda_{00}(q)=0, \\
-i \frac{q_{i} q_{0}}{\vec{q}^{2}} D(q)+D_{i 0}(q)-\frac{q_{j} q_{0}}{\vec{q}^{2}} D_{i j}(q)-\frac{g}{\vec{q}^{2}} \Lambda_{i 0}(q)=0,
\end{array}
$$

where

$$
\begin{aligned}
D(q) & =\int d x e^{i q \cdot x}<0|T c(x) \bar{c}(0)| 0>, \\
D_{\mu \nu}(q) & =\int d x e^{i q \cdot x}<0\left|T A_{\mu}(x) A_{\nu}(0)\right| 0>, \\
\Lambda_{\mu \nu}(q) & =\int d x e^{i q \cdot x}<0\left|T A_{\mu}(x) J_{\nu}(0)\right| 0>.
\end{aligned}
$$

If one divides $D_{\mu \nu}(q)$ as

$$
D_{\mu \nu}(q)=D_{\mu \nu}^{(0)}(q)+\tilde{D}_{\mu \nu}(q)
$$

where $D_{\mu \nu}^{(0)}(q)$ is a propagator of gauge field at the tree level which can be obtained from usual Feynman rule and $\tilde{D}_{\mu \nu}(q)$ is a higher order correction, Eq.(10) becomes for n-loop order

$$
\begin{array}{rllll}
-i D(q)+D_{00}^{(0)}(q) & =0 & \text { for } & n=0, \\
-i \frac{q_{i} q_{0}}{\vec{q}^{2}} D(q)-\frac{q_{j} q_{0}}{\vec{q}^{2}} D_{i j}^{(0)}(q) & = & 0 & \text { for } & n=0, \\
\tilde{D}_{00}(q)-\frac{q_{i} q_{0}}{\vec{q}^{2}} \tilde{D}_{0 i}(q)-\frac{g}{\vec{q}^{2}} \Lambda_{00}(q) & =0 & \text { for } & n \geq 1, \\
\tilde{D}_{i 0}(q)-\frac{q_{j} q_{0}}{\vec{q}^{2}} \tilde{D}_{i j}(q)-\frac{g}{\vec{q}^{2}} \Lambda_{i 0}(q) & =0 & \text { for } & n \geq 1 .
\end{array}
$$


One can show easily that the first two equations of Eq.(13) become

$$
q^{\mu} D_{\mu \nu}^{(0)}(q)=i q_{\nu} D(q)
$$

By using the trivial identity

$$
\Lambda_{\mu \nu}(q)=-\frac{q^{2}}{g} \tilde{D}_{\mu \nu}(q)
$$

which is valid in $\mathrm{n}(\geq 1)$-loop level and can be easily proved by Feynman diagram, the last two equations of Eq.(13) become

$$
q^{\mu} \tilde{D}_{\mu \nu}=0
$$

Fig.(1) shows the example of trivial identity (15) at one-loop level. Combining (14) and (16) one can obtain WT identity

$$
q^{\mu} D_{\mu \nu}(q)=i q_{\nu} D(q)
$$

Eq.(17) is well-known WT identity which is derived from the usual BRST symmetry. So in this case LM symmetry does not produce new WT identity. Now let us examine the WT identity which contains the vertex. For this let us start with a identity of QED

$$
<0|T \psi(x) \bar{\psi}(y) c(z)| 0>=0
$$

By following the same procedure LM symmetry provides

$$
\begin{array}{r}
i g \frac{q_{0}}{\vec{q}^{2}} D(q)\left[S_{F}(p+q)-S_{F}(p)\right]+\Gamma_{0}(p, q,-p-q)- \\
\frac{q_{i} q_{0}}{\vec{q}^{2}} \Gamma_{i}(p, q,-p-q)-\frac{g}{\vec{q}^{2}} K_{0}(p, q,-p-q)=0
\end{array}
$$

where

$$
\begin{aligned}
S_{F}(q) & =\int d x e^{i q \cdot x}<0|T \psi(x) \bar{\psi}(0)| 0>, \\
<0\left|T \psi(x) A_{\mu}(z) \bar{\psi}(y)\right| 0> & =\int d p d q d r \Gamma_{\mu}(p, q, r) e^{-i p \cdot x-i q \cdot z-i r \cdot y} \delta(p+q+r), \\
<0\left|T \psi(x) J_{\mu}(z) \bar{\psi}(y)\right| 0> & =\int d p d q d r K_{\mu}(p, q, r) e^{-i p \cdot x-i q \cdot z-i r \cdot y} \delta(p+q+r) .
\end{aligned}
$$


In Eq.(20) an energy-momentum conserving delta function is included in the vertex-type objects. From Fig.(2) one can prove straightforwardly the trivial identity

$$
K_{\mu}(p, q,-p-q)=-\frac{q^{2}}{g} \Gamma_{\mu}(p, q,-p-q) .
$$

By using Eq.(21) the WT identity

$$
q^{\mu} \Gamma_{\mu}(p, q,-p-q)=-i g D(q)\left[S_{F}(p+q)-S_{F}(p)\right]
$$

is straightforwardly derived from Eq.(19). The WT identity (22) can be easily re-derived from the BRST symmetry and gives rise to a famous formula

$$
Z_{1}=Z_{2}
$$

by the Dyson Z-notation.So LM symmetry does not give any new non-trivial identity in this case too.

Being inspired by the examination of WT identities, we can construct the generalized symmetries of QED

$$
\begin{aligned}
\delta_{G} A_{0} & =i \bar{c} \\
\delta_{G} A_{i} & =-\partial_{i} a(-i \partial) \bar{c} \\
\delta_{G} \bar{c} & =0 \\
\delta_{G} c & =A_{0}-i \partial_{i} a(-i \partial) A_{i}+b(-i \partial) J_{0}-i \partial_{i} f(-i \partial) J_{i}, \\
\delta_{G} \psi & =(g(-i \partial) \bar{c}) \psi, \\
\delta_{G} \bar{\psi} & =\bar{\psi}(g(-i \partial) \bar{c}),
\end{aligned}
$$

where $a(x), b(x), f(x)$ and $g(x)$ are functions of four vector. If one requires the conditions

$$
x_{0} a(x)-\frac{x^{2}}{g}\left[x_{0} f(x)+b(x)\right]=-1,
$$




$$
\begin{aligned}
g(x) & =i g\left[a(x)-\frac{x^{2}}{g} f(x)\right], \\
a(-x) & =-a(x), \\
b(-x) & =b(x), \\
f(-x) & =-f(x),
\end{aligned}
$$

it is straightforward to prove that the transformation(24) with restrictions (25) is symmetry for the QED Lagrangian(2). Of course LM transformation is a special case of $(24)$ :

$$
\begin{aligned}
& a_{L M}(x)=-\frac{x_{0}}{\vec{x}^{2}}, \\
& b_{L M}(x)=-\frac{g}{\vec{x}^{2}}, \\
& f_{L M}(x)=0, \\
& g_{L M}(x)=-i g \frac{x_{0}}{\vec{x}^{2}} .
\end{aligned}
$$

By the same procedure one can show easily that this generalized symme$\operatorname{try}(24)$ with (25) provides same WT identities (17) and (22) using the trivial identities(15) and (21).

In conclusion although LM transformation is non-local and non-covariant symmetry, not only LM symmetry but also the generalized symmetry does not yield any new non-trivial identity at quantum level.

\section{References}

[1] M.Lavelle and D.McMullan, Phys. Rev. Lett. 71, 3758(1993).

[2] C.Becchi, A.Rouet and R.Stora, Phys. Lett. B52, 344(1974).

[3] I.V.Tyutin, Lebedev preprint, FIAN No.39(1975), unpublished. 
[4] Victor O.Rivelles, hep-th9509028.

[5] M.Lavelle and D.McMullan, hep-th9509159. 


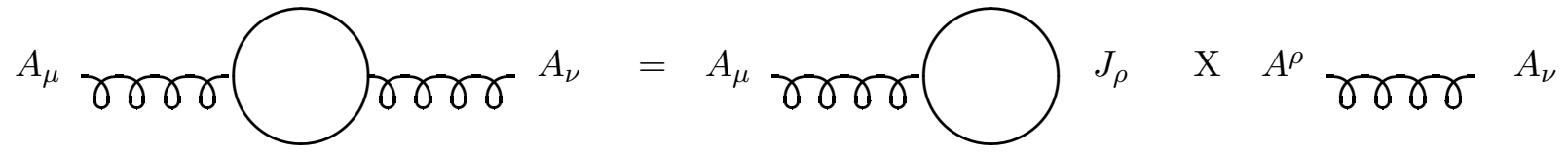

Figure 1: one-loop example of trivial identity(15)

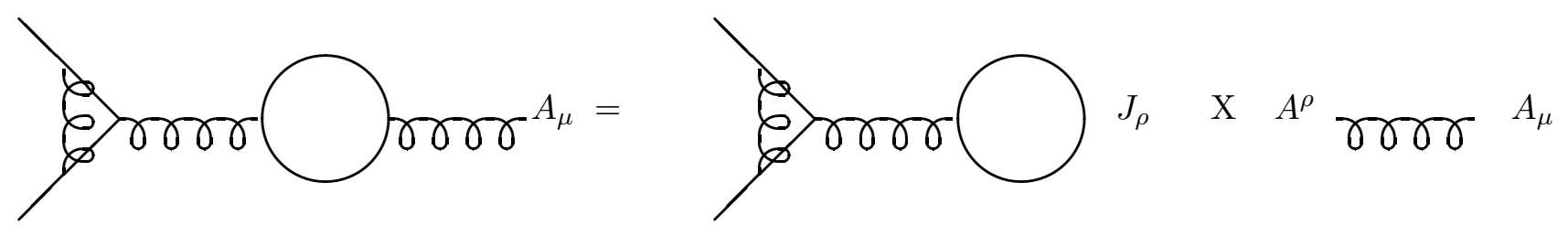

Figure 2: two-loop example of trivial identity(21) 